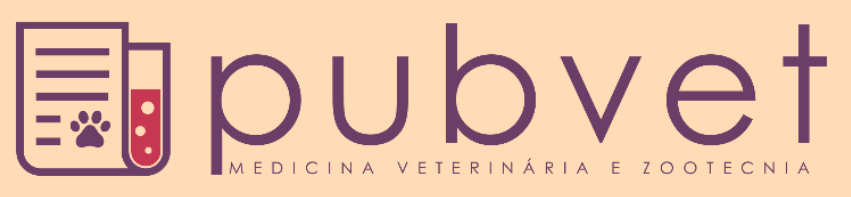

https://doi.org/10.31533/pubvet.v13n10a436.1-4

\title{
Diagnóstico de Taenia taeniaeformis (Cyclophyllidea: Taeniidae) em felino doméstico em Pelotas, RS: relato de caso
}

\author{
Julia Somavilla Lignon $^{1 *}$, Tatiana de Ávila Antunes ${ }^{2} \bullet$, Vinícius Macedo Padilha ${ }^{3} \bullet$, Marcelo \\ Dantas Ritta $^{3}{ }^{\circ}$, Hellen Mariane Dantas ${ }^{4}$, Diego Moscarelli Pinto ${ }^{5}$ \\ ${ }^{1}$ Médica Veterinária, Departamento de Veterinária Preventiva, Universidade Federal de Pelotas - UFPel, Pelotas, RS, Brasil. \\ ${ }^{2}$ Médica Veterinária, Residente em Doenças e Zoonoses Parasitárias, Departamento de Veterinária Preventiva, UFPel, Pelotas, RS, Brasil. \\ ${ }^{3}$ Médico Veterinário, Universidade Federal de Pelotas, Pelotas, RS, Brasil. \\ ${ }^{4}$ Graduanda em Medicina Veterinária, Universidade Federal de Pelotas, Pelotas, RS, Brasil \\ ${ }^{5}$ Médico Veterinário, Professor na disciplina de Doenças Parasitárias, Departamento de Veterinária Preventiva, UFPel, Pelotas, RS, Brasil. \\ *Autor para correspondência, E-mail: julialignon@gmail.com
}

\begin{abstract}
Resumo. A Teníase causada pelo cestoda Taenia taeniaeformis, é uma enfermidade cosmopolita que acomete o intestino delgado dos felinos, sendo considerados hospedeiros definitivos. Os hospedeiros intermediários são diversos roedores, causando-lhes alterações estruturais acompanhadas de disfunção das células do fígado. Em geral, as infecções são consideradas assintomáticas, no entanto já foi relatado obstrução intestinal por esse parasito. Um caso de infecção humana já foi relatado. O presente relato descreve um caso de $T$. taeniaeformis diagnosticado em um gato atendido em uma clínica veterinária em Pelotas, RS, Brasil. É o primeiro caso relatado com sinais clínicos na região, além disso, não há relatos recentes sobre sua ocorrência no país. Utilizou-se a associação comercial dos princípios ativos Pamoato de pirantel $(20 \mathrm{mg} / \mathrm{Kg})$ e Praziquantel $(5 \mathrm{mg} / \mathrm{Kg})$. Este trabalho deve servir como alerta para Médicos Veterinários de toda a região, pois existe a possibilidade de risco clínico da teníase em gatos e a possível ocorrência em humanos.
\end{abstract}

Palavras chave: cestódeo, roedores, teníase

\section{Diagnosis of Taenia taeniaeformis (Cyclophyllidea: Taeniidae) in domestic feline in Pelotas city, Brazil: case report}

\begin{abstract}
Taeniasis, caused by the Taenia taeniaeformis cestoda, is a cosmopolitan disease affecting the feline small intestine and is considered a definitive host. Intermediate hosts are several rodents, causing structural changes accompanied by liver cell dysfunction. In general, infections are considered asymptomatic, however intestinal obstruction has been reported by this parasite. A case of human infection has already been reported. The present report describes a case of $T$. taeniaeformis diagnosed in a cat seen at a veterinary clinic in Pelotas, RS, Brazil. It is the first case reported with clinical signs in the region, and there are no recent reports of its occurrence in the country. The commercial association of the active ingredients Pamoato de pirantel $(20 \mathrm{mg} / \mathrm{Kg})$ and Praziquantel $(5 \mathrm{mg} / \mathrm{Kg})$ was used. This work should serve as a warning to veterinarians throughout the region, as there is a potential clinical risk of teniasis in cats and possible occurrence in humans.
\end{abstract}

Keywords: cestode, rodents, teniasis

\section{Diagnóstico de Taenia taeniaeformis (Cyclophyllidea: Taeniidae) en felino doméstico en la ciudad de Pelotas,Brasil: reporte de un caso}

Resumen. La teniasis, causada por Taenia taeniaeformis cestoda, es una enfermedad cosmopolita que afecta al intestino delgado felino y se considera un huésped definitivo. 
Los hospedadores intermedios son varios roedores, que causan cambios estructurales acompañados de disfunción de las células hepáticas. En general, las infecciones se consideran asintomáticas, sin embargo, ya fue reportada la obstrucción intestinal causada por este parasito. Un caso de infección humana ya fue reportado. El presente informe describe un caso de $T$. taeniaeformis diagnosticada en un gato visto en una clínica veterinaria en Pelotas, RS, Brasil. Es el primer caso reportado con signos clínicos en la región, y no hay informes recientes de su ocurrencia en el país. Se usó la asociación comercial de los ingredientes activos Pamoato de pirantel $(20 \mathrm{mg} / \mathrm{Kg})$ y Praziquantel $(5 \mathrm{mg} / \mathrm{Kg})$. Este trabajo debería servir como una advertencia para los veterinarios de toda la región, ya que existe un riesgo clínico potencial de teniasis en gatos y una posible aparición en humanos.

Palabras clave: cestodo, roedores, teniasis

\section{Introdução}

A Teníase causada pelo cestoda Taenia taeniaeformis, é uma enfermidade cosmopolita que acomete o intestino delgado dos felinos e, ocasionalmente, cães, sendo esses considerados hospedeiros definitivos (Fortes, 2004; Monteiro et al., 2017; Taylor et al., 2017).

Os gatos eliminam proglótides de T. taeniaeformis cerca de 20 dias após a ingestão da forma larval Cysticercus fasciolaris encontrados no fígado e cavidade peritoneal de roedores infectados, como ratos, camundongos, coelhos e esquilos, sendo esses os hospedeiros intermediários (Fortes, 2004; Hutchison, 1957; Taylor et al., 2017). Os hospedeiros intermediários são infectados ao ingerirem pastos contaminados e apresentam ovos de T. taeniaeformis. Os ovos embrionados eclodem no intestino delgado e larvas migram através do sangue portal para o fígado, onde formam cistos. As larvas raramente migram para outros órgãos, mas causam alterações estruturais acompanhadas de disfunção das células do fígado. Geralmente, a forma larval encontra-se envolvida por uma reação de neoformação sarcomatosa (Monteiro et al., 2017). Em geral, cestódeos adultos, que medem cerca de 15 a $60 \mathrm{~cm}$ de comprimento, têm pouca significância patogênica e as infecções são consideradas assintomáticas (Fortes, 2004; Monteiro et al., 2017; Taylor et al., 2017). No entanto, Wilcox et al. (2009) relataram obstrução intestinal por esse parasito.

Um único caso de infecção humana com um estágio larval de T. taeniaeformis foi relatado (Stěrba \& Barus, 1976). Neste caso, um homem de 77 anos morreu de causas não relacionadas e a necropsia revelou que havia numerosos cistos serosos no fígado, onde continham a forma larval. Com base na prevalência relativamente alta de $T$. taeniaeformis em todo o mundo, o fato de haver apenas uma única infecção humana com essa fase do parasito indica que ele não se desenvolve bem no hospedeiro humano, não descartando sua ocorrência.

$\mathrm{O}$ presente relato descreve um caso de $T$. taeniaeformis diagnosticado em um gato atendido em uma clínica veterinária particular da cidade de Pelotas, no estado do Rio Grande do Sul, Brasil.

\section{Relato de caso}

Um felino, macho, SRD, não castrado, semi domiciliado, com dois anos de idade, pesando cerca de 3,4 kg e alimentado com ração foi encaminhado para internação em uma clínica veterinária localizada na cidade de Pelotas, no Rio Grande do Sul. A tutora relatou que o animal apresentava falta de apetite e estava com inapetência há três dias, além disso, apresentava hematoquezia. No exame físico e clínico observou-se perda de peso, desidratação e algia abdominal, não apresentando febre e parâmetros como frequência cardíaca e respiratória dentro dos parâmetros fisiológicos para a espécie. O animal havia sido vermifugado pela última vez seis meses antes da consulta e o controle de ectoparasitos e vacinação estava atrasado. Segundo o relato do tutor, o felino possuía mais sete contactantes, que não apresentavam os sinais clínicos mencionados. Os animais possuíam livre acesso à rua apresentando contato com roedores e outros animais.

No momento da internação, realizou-se fluidoterapia com adição de Complexo B e Glicose 50\%. Além disso administrou-se Dipirona ( $25 \mathrm{mg} / \mathrm{kg})$ e Metronidazol $(15 \mathrm{mg} / \mathrm{kg})$. 
Durante a internação, o veterinário presenciou a eliminação de parasitos cestódeos nas fezes do paciente, além de hematoquezia. Portanto, utilizou-se a associação comercial dos princípios ativos Pamoato de pirantel $(20 \mathrm{mg} / \mathrm{kg})$ e Praziquantel $(5 \mathrm{mg} / \mathrm{kg})$, um vermífugo oral de dose única de amplo espectro para gatos, sendo administrado um comprimido. Após a realização do tratamento, houve mais eliminação de parasitos pelas fezes. Os mesmos foram armazenados em álcool $70 \%$ e encaminhados para identificação da espécie no laboratório do Grupo de Estudos em Enfermidades Parasitárias na Faculdade de Veterinária da Universidade Federal de Pelotas.

O parasito, medindo $40 \mathrm{~cm}$ de comprimento, foi identificado pela maceração de seus proglótides maduros, apresentando morfologia dos ovos (Figura 1) e proglótides compatíveis com Taenia taeniaeformis.

No dia seguinte a internação, solicitou-se a realização de exame complementar como hemograma. $\mathrm{O}$ Eritrograma e Leucograma apresentaram-se dentro dos limites fisiológicos. Após a administração do vermífugo, da hidratação e do tratamento de suporte, o paciente reestabeleceu o apetite e voltou a se alimentar sozinho.

O paciente não voltou à clínica para a consulta de retorno, não sendo possível realizar a avaliação da eficácia da associação dos princípios ativos utilizados no tratamento.

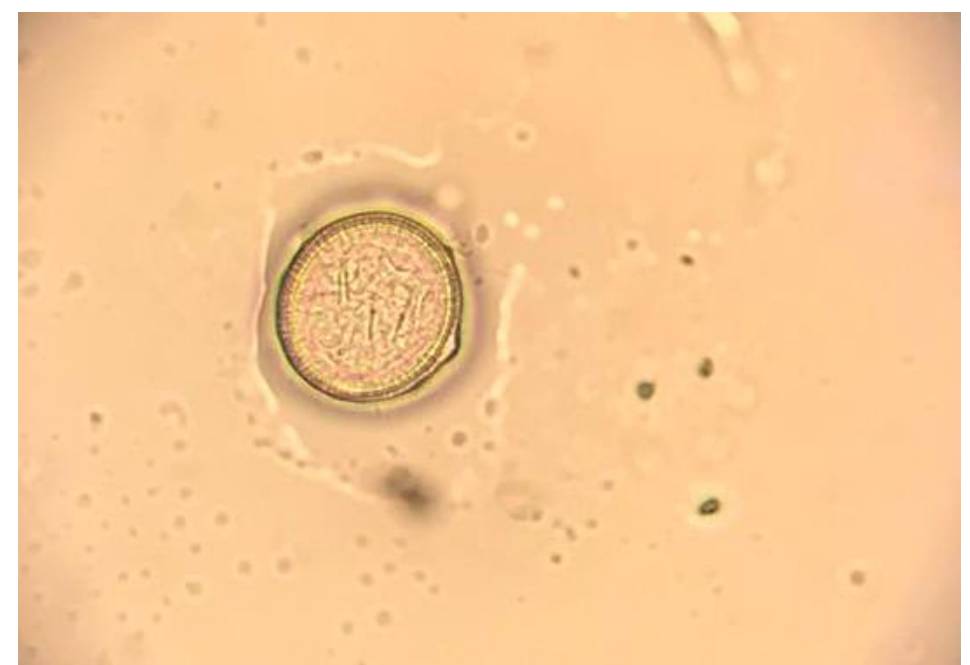

Figura 1. Ovo de Taenia taeniaeformis com embrião hexacanto obtido após maceração de proglótides grávidos.

\section{Discussão}

Embora T. taeniaeformis tenha distribuição mundial, existem poucos relatos, sobre a incidência da tênia adulta. No Brasil, não há relatos recentes sobre sua ocorrência, no entanto, pode haver infecções assintomáticas não diagnosticadas, levando em consideração que as infecções não apresentam sinais clínicos evidentes (Taylor et al., 2017). Também não foram publicados casos que descrevam doença ou sinais deletérios de infecção associados a tênias adultas no trato intestinal do hospedeiro felino. Geralmente, em gatos assim como em humanos, os casos de teníase intestinal são bem tolerados (Wilcox et al., 2009).

Em 2009, o primeiro relato com sinais clínicos foi diagnosticado por Wilcox et al. (2009), onde houve obstrução intestinal por esse parasito em um felino doméstico, castrado, cinco meses de idade, $6,3 \mathrm{~kg}$, apresentando histórico de vômitos agudos, anorexia, letargia e dispneia. O paciente era um "gato de celeiro", sem histórico de vacinação e administração anti-helmíntica. O gato respondeu com sucesso à intervenção cirúrgica com múltiplas enterotomias e remoção de cestodeos inteiros e seus segmentos do intestino delgado. Além disso, com administração trimestral de Praziquantel $(5 \mathrm{mg} / \mathrm{kg})$ via oral, não foi observada recorrência adicional de sinais clínicos. Da mesma forma, o presente relato apresentou sinais clínicos como algia abdominal, hematoquezia, desidratação e perda de peso.

Hutchison (1957) avaliou a incidência de T. taeniaeformis em gatos na Escócia, sendo encontrada em 132 animais, obtendo incidência de 7,7\%. Uma avaliação parasitológica realizada por Jacinto (2016), 
durante os meses de setembro de 2015 a março de 2016, em Lisboa, Portugal, obteve prevalência de 3,3\% para T. taeniaeformis. Hasanpour et al. (2017), em Kermanshah no Irã, encontraram uma prevalência de 4,3\% de Cysticercus fasciolaris, a forma larval da T. taeniaeformis, em roedores. A maior prevalência de infecções por cestodeos foi relatado em felinos domésticos na Europa, com 33\% dos gatos infectados com T. taeniaeformis na Áustria. No Brasil, Mundim et al. (2004) avaliaram a frequência de helmintos em gatos necropsiados no município de Uberlância em Minas Gerais, onde T. taeniaeformis apresentou frequência de 4\%. No município de Goiânia, em Goiás, Campos et al. (1974) encontraram prevalência de $3 \%$ para $T$. taeniaeformis.

Segundo Jacinto (2016), o Praziquantel e o Epsiprantel demonstraram ser eficazes no tratamento da T. taeniaeformis em gatos. Da mesma forma, o Febantel e o Fenbendazol são aprovados para o tratamento deste parasita na maior parte do mundo. Mebendazol e Nitroscanato também são produtos que têm eficácia contra esses parasitos em gatos.

\section{Conclusão}

O presente trabalho é o primeiro relato de Teníase por T. taeniaeformis na cidade de Pelotas, Rio Grande do Sul. Embora o parasito adulto raramente cause doença clínica em gatos, este relato indica que a vigilância deve ser empregada em pacientes felinos, principalmente àqueles em risco com histórico de acesso a roedores e nenhum tratamento regular para cestódeos. É importante, portanto, que os tutores levem os felinos ao veterinário anualmente para realização de um check-up e façam exames laboratoriais, incluindo um exame fecal. A prevenção rotineira deve ser realizada, e este trabalho deve servir como alerta para Médicos Veterinários de toda a região, pois existe a possibilidade de risco clínico da teníase em gatos e a possível ocorrência em humanos.

\section{Referências bibliográficas}

Campos, D. B., Garibaldi, I. M. \& Carneiro, J. R. (1974). Prevalência de helmintos em gatos (Felis catus domesticus) de Goiânia. Revista de Patologia Tropical, 3(4):355-359.

Fortes, E. (2004). Parasitologia veterinária (4 ed.). São Paulo: Editora ícone.

Hasanpour, H., Najafi, F., Gharagozlou, M. J., Jafarpour Azami, S., Fadavi, A., Paknezhad, N. \& Mowlavi, G. (2017). Cysticercus fasciolaris (Taenia taeniaeformis Larval Stage) in urban rats with illustration of histopathological changes in the liver. Journal of Medical Microbiology and Infectious Diseases, 5(3):43-46.

Hutchison, W. M. (1957). The incidence and distribution of Hydatigera taeniaeformis and other intestinal helminths in scottish cats. Journal of Parasitology, 43(3):318-383.

Jacinto, A. G. (2016). Rastreio parasitológico de Tritrichomonas foetus em gatos. Master of Science, Universidade de Lisboa, Lisboa, Portugal.

Monteiro, M. J. d. S. D., Santos, G. M., Barreto, M. T. S., Silva, R. V. S., Jesus, R. L. R. \& Silva, H. J. N. (2017). Perfil epidemiológico de casos de hanseníase em um estado do nordeste brasileiro. Revista de Atenção à Saúde, 15(54):21-28.

Mundim, T. C. D., Oliveira Júnior, S. D., Rodrigues, D. C. \& Cury, M. C. (2004). Frequency of helminthes parasites in cats of Uberlândia, Minas Gerais. Arquivo Brasileiro de Medicina Veterinária e Zootecnia, 56(4):562-563.

Stěrba, J. \& Barus, V. (1976). First record of Strobilocercus fasciolaris (Taenidae-larvae) in man. Folia Parasitologica, 23(3):221-226.

Taylor, M. A., Coop, R. L. \& Wall, R. L. (2017). Parasitologia Veterinária. Rio de Janeiro, Brasil: Guanabara Koogan.

Wilcox, R. S., Bowman, D. D., Barr, S. C. \& Euclid, J. M. (2009). Intestinal obstruction caused by Taenia taeniaeformis infection in a cat. Journal of the American Animal Hospital Association, 45(2):93-96.

Recebido: 30 de agosto, 2019.

Aprovado: 6 de novembro, 2019.

Publicado: 3 de dezembro, 2019.

Licenciamento: Este artigo é publicado na modalidade Acesso Aberto sob a licença Creative Commons Atribuição 4.0 (CC-BY 4.0), a qual permite uso irrestrito, distribuição, reprodução em qualquer meio, desde que o autor e a fonte sejam devidamente creditados. 